\title{
On the LQG Control for a Class of Nonlinear Stochastic Systems-Simulation Studies
}

\author{
Y. Sunahara*, H. Ohtagaki** and OT. Tsutsumi* \\ *) Department of Mechanical Engineering, Okayama University of Science, \\ **) Department of Electronic Engineering, Okayama University of Science, \\ Ridai-cho, Okayama 700 Japan
}

In this paper, under the LQG control performance criterion, a method for realizing the suboptimal control for one-dimensional nonlinear stochastic systems with finite horizon is developed by exhibiting a wide variety of sample runs.

As preliminaries, the equation of determining state trajectory satisfying the performance criterion and its linearization scheme are reviewed. In order to realize finite horizon control for nonlinear systems, the suboptimal state trajectory emanating from the initial state is switched at time $t_{s}$ into the one hitting the target. Decision of switching time is illustrated by results of simulation experiments.

Finally, relative relation between the initial state level and the target state one is examined.

\section{INTRODUCTION}

Mathematical aspects of the infinite horizon optimal control were studied in Refs. [1] and [2]. However, in the finite horizon optimal control problem of nonlinear stochastic systems, no feasible methods may be found because many difficulties arise in both theoretical and computational aspects for realizing the suboptimal control strategy.

The key idea of the method presented is to revive a diverging process caused by the unstable strategy and to hit the fixed target in the stochastic sense. Throughout the paper, a class of the onedimensional nonlinear stochastic systems is considered under the LQG performance criterion.

In Sec. 2, necessary formulas are exhibited for explaining simulation studies where some of them is briefly illustrated with respect to their derivations, in Appendices.

In Sec. 3, introducing a linearization technique, the one-dimensional difference formula implemented by a recurrent computation is derived for determining the suboptimal state trajectory.

In Sec. 4, detailed aspects of performing simulation experiments and of realizing the suboptimal state trajectories are explained by showing representative sample runs of the state trajectories.

Section 5 concludes the study presented here.

\section{PRELIMINARIES}

The system of concern is given by the one-dimensional stochastic nonlinear system,

$$
\begin{aligned}
& d x=\{f(x)+b u\} d t+\sigma_{0} x d w, \\
& x(0)=x_{0}, \quad(0 \leq t<T),
\end{aligned}
$$

where $x$ is the system state, $u$ is the control, $f(x)$ is bounded and $f(0)=0, b$ and $\sigma_{0}$ are constant parameters, and $w$ is the standard Wiener process, respectively. We assume that there exists a continuous solution to Eq. (1).

Given $x_{0}$, the problem is to find the optimal control so as to satisfy the performance criterion,

$$
J(u)=\min _{u \in \mathcal{U}^{0}}\left[E_{x_{0}}\left\{\int_{0}^{T}\left(m x_{t}^{2}+n u_{t}^{2}\right) d t\right\}\right],
$$

and $E_{x_{0}}\{x(T)\}=x_{T}$, where $E_{x_{0}}\{*\}$ represents the mathematical expectation conditioned by $x_{0}, \mathcal{U}^{0}$ is a family of admissible controls, $m$ and $n$ are constants where $n \neq 0$.

Let the value function $V$ be

$$
V\left(t, x_{t}\right)=\min _{u \in \mathcal{U}^{0}}\left[E_{x_{t}}\left\{\int_{t}^{T}\left(m x_{s}^{2}+n u_{s}^{2}\right) d s\right\}\right],
$$

Then, the Hamilton-Jacobi-Bellman equation is

$$
\min _{u \in \mathcal{U}^{0}}\left[m x_{t}^{2}+n u_{t}^{2}+\left\{f\left(x_{t}\right)+b u_{t}\right\} V_{x_{t}}\right.
$$




$$
\left.+\frac{1}{2} \sigma_{0}^{2} x_{t}^{2} V_{x_{i} x_{i}}\right]=-V_{t}
$$

where $V_{*}$ and $V_{* *}$ denote partial derivatives of the function $V$, respectively. We may define formally the function $K$ by

$$
\begin{aligned}
& K\left(x_{t}, u_{t}, V_{x_{t}}, V_{x_{i} x_{t}}\right) \\
& \quad=m x_{t}^{2}+n u_{t}^{2}+\left\{f\left(x_{t}\right)+b u_{t}\right\} V_{x_{t}}+\frac{1}{2} \sigma_{0}^{2} x_{t}^{2} V_{x_{t} x_{t}} .
\end{aligned}
$$

In the LQG control scheme, the equation for determining the steady state of the optimally controlled system is

$$
\frac{n}{b^{2}} f(\vec{x}) \frac{\partial f(\vec{x})}{\partial \vec{x}}+\sigma_{0}^{2} p_{s} \vec{x}+m \vec{x}=0,
$$

where $p_{s}$ is the stationary solution of Riccati equation which is shown and $\bar{x}$ is the steady state of the system (1) (see Appendix-A). The state trajectory of the system (1) satisfying the performance criterion (2) is determined by (see Appendix-B)

$$
\begin{aligned}
& \left(d x_{t}^{*}-\sigma_{0} x_{t}^{*} d w\right)^{2} \\
& =\frac{b^{2}}{n}\left\{m\left(x_{t}^{*^{2}}-\bar{x}^{2}\right)\right. \\
& \left.\quad+\frac{n}{b^{2}}\left(f^{2}\left(x_{t}^{*}\right)-f^{2}(\bar{x})\right)+\sigma_{0}^{2} p_{s}\left(x_{t}^{*^{2}}-\bar{x}^{2}\right)\right\} d t^{2} .
\end{aligned}
$$

\section{APPROXIMATION AND SUBOPTI-}

\section{MAL STATE TRAJECTORY}

In order to solve Eqs. (6) and (7) simultaneously, we consider the time partition such that $t=k \Delta t(k \Delta t \leq t<\infty)$, where $k=0,1,2, \cdots$, and expanding the nonlinear function $f(x)$ into

$$
f\left(x_{t}^{*}\right)=a_{k}^{*} x_{t}^{*}+c_{k}^{*} \quad(k \Delta t \leq t<\infty),
$$

where

$$
\begin{aligned}
& a_{k}^{*}=f_{x_{k}^{*}}\left(x_{k}^{*}\right), \quad c_{k}^{*}=f\left(x_{k}^{*}\right)-f_{x_{k}^{*}}\left(x_{k}^{*}\right) x_{k}^{*}, \\
& f_{x_{k}^{*}}\left(x_{k}^{*}\right)=\frac{\partial f\left(x_{k}^{*}\right)}{\partial x_{k}^{*}} .
\end{aligned}
$$

By using Eq. (8), Eqs. (6) and (7) are linearized as

$$
\vec{x}_{s k}=\frac{-n a_{k}^{*} c_{k}^{*}}{b^{2} m+a_{k}^{* 2} n+b^{2} \sigma_{0}^{2} p_{s k}}
$$

and

$$
\begin{aligned}
& \left(d x_{t}^{*}-\sigma_{0} x_{t}^{*} d w\right)^{2} \\
& =\frac{b^{2}}{n}\left\{m+\frac{n}{b^{2}} a_{k}^{*}+\sigma_{0}^{2} p_{s k}\right\}\left\{\left(x_{t}^{*}-\bar{x}_{s k}\right) d t\right\}^{2} \\
& \text { for } k \Delta t \leq t<\infty,
\end{aligned}
$$

respectively, where in Eq. (3), as $T \rightarrow \infty, V_{x_{i}^{*} x_{i}^{*}} \simeq$ $V_{\overrightarrow{x x}}=2 p_{s k}$ (Ref. [2]), and $p_{s k}$ is the solution to the linearized algebraic Riccati equation,

$$
\frac{b^{2}}{n} p_{s k}^{2}-\left(2 a_{k}^{*}+\sigma_{0}^{2}\right) p_{s k}-m=0
$$

The solutions to Eq. (12) are

$$
\begin{aligned}
p_{s k}^{+} & , \overline{p_{s k}^{-}} \\
= & \frac{n\left(2 a_{k}^{*}+\sigma_{0}^{2}\right) \pm \sqrt{n^{2}\left(2 a_{k}^{*}+\sigma_{0}^{2}\right)^{2}+4 b^{2} m n}}{2 b^{2}} .
\end{aligned}
$$

Equation (11) is decomposed into

$$
\begin{array}{r}
d x_{t}^{*-}=-\sqrt{\phi_{k}}\left(x_{t}^{*-}-\bar{x}_{s k}\right) d t+\sigma_{0} x_{t}^{*-} d w_{t} \\
\text { for } k \Delta t \leq t<\infty,
\end{array}
$$

and

$$
\begin{array}{r}
d x_{t}^{*+}=+\sqrt{\phi_{k}}\left(x_{t}^{*+}-\bar{x}_{s k}\right) d t+\sigma_{0} x_{t}^{*+} d w_{t} \\
\text { for } k \Delta t \leq t<\infty,
\end{array}
$$

where

$$
\phi_{k}=n^{-1}\left[b^{2}\left(m+\sigma_{0}^{2} p_{s k}\right)+n a_{k}^{* 2}\right] .
$$

First, suppose that $x=0$ and as shown in Fig. 1 , determine the switching time $t_{s}$ by $\bar{x}_{d}^{*-}=\bar{x}_{d}^{*+}$, where $\bar{x}_{d}^{*-}$ and $\bar{x}_{d}^{*+}$ are the ensemble averaged processes and, without destination of $x_{0}>0$ or $x_{0}<$ 0 , these are the solutions to

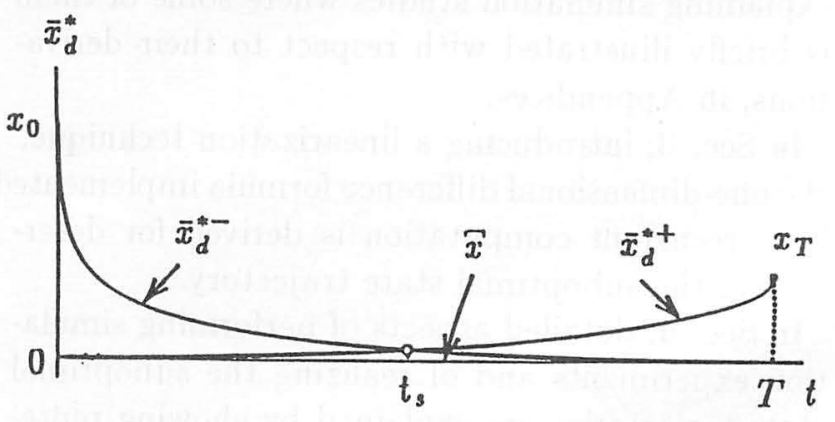

Fig. 1: Schematic illustration of the switching time $t_{s}$ 


$$
\begin{gathered}
\dot{\bar{x}}_{d}^{*-}=-\sqrt{\phi_{k}}\left(\bar{x}_{d}^{*-}-\bar{x}_{s k}^{-}\right) \\
\text {for } k \Delta t \leq t<\infty,
\end{gathered}
$$

and

$$
\begin{gathered}
\dot{\bar{x}}_{d}^{*+}=+\sqrt{\phi_{k}}\left(\bar{x}_{d}^{*+}-\bar{x}_{s k}^{+}\right) \\
\text {for } k \Delta t \leq t<\infty .
\end{gathered}
$$

where from (10), we write $\mathscr{x}_{s k}^{-}, \bar{x}_{s k}^{+}$for $\widetilde{x}_{s k}^{-}=\left.\breve{x}_{s k}\right|_{p_{s k}=p_{s k}^{-}}$ , $\bar{x}_{s k}^{+}=\left.\bar{x}_{s k}\right|_{p_{s k}=p_{s k}^{+}}$, respectively.

Secondly, Eq. (11) is decomposed into

$$
\begin{array}{r}
d x_{t}^{*-}=-\sqrt{\phi_{k}^{-}}\left(x_{t}^{*-}-\bar{x}_{s k}^{-}\right) d t+\sigma_{0} x_{t}^{*-} d w_{t} \\
\text { for } \quad k \Delta t \leq t<t_{s}=k_{s} \Delta t,
\end{array}
$$

and

$$
\begin{array}{r}
d x_{t}^{*+}=+\sqrt{\phi_{k}^{+}}\left(x_{t}^{*+}-\vec{x}_{s k}^{+}\right) d t+\sigma_{0} x_{t}^{*+} d w_{t} \\
\text { for } \quad t_{s}=k_{s} \Delta t<t \leq T .
\end{array}
$$

where we write $\phi_{k}^{-}, \phi_{k}^{+}$for $\phi_{k}^{-}=\left.\phi_{k}\right|_{p_{s k}=p_{s k}^{-}}, \phi_{k}^{+}=$ $\left.\phi_{k}\right|_{p_{s k}=p_{s k}^{+}}$. Start with the given initial state $x_{0}$ and the sample process $x_{t}^{*-}$ runs along Eq. (19) to the switching time $t_{s}$.

At time $t_{s}$ the sample process $x_{t}^{*-}$ is switch into the $x_{t}^{*+}$-process and this runs along Eq. (20) to the terminal time $\mathrm{T}$.

\section{SIMULATED RUNS}

The nonlinear function of concern is

$$
f(x)=\alpha x-\beta x^{3}, \quad(\alpha, \beta>0) .
$$

In Eqs. (1) and (21), the values of the parameters were

$$
\alpha=1, \quad \beta=1, \quad b=4, \quad \sigma_{0}=0.3
$$

and setting as $m=1, n=1$ and $T=12$, the performance criterion given by (2) was written by

$$
J(u)=\min _{u \in \mathcal{U}^{0}}\left[E_{x_{0}}\left\{\int_{0}^{12}\left(x_{t}^{2}+u_{t}^{2}\right) d t\right\}\right] .
$$

It is natural that there exist many values of $\bar{x}$ satisfying Eq. (6). However, in the light of Eq. (21), $\vec{x}=0$ is chosen among five values of $\vec{x}$ obtained by Eq. (6). It should be noted that the application of this choice is limited to the preassigned range of initial sates which will be explained in the subsequent paper. Thus, omitting to explain the situation of initial states, we set as $x_{0}=1$ which is allowed to choose the value of $\bar{x}=0$.

First, Eqs. (17) and (18) were simulated a digital computer, Along the line of procedures described in the previous section, the switching time $t_{s}$ may be determined. Table 1 and Fig. 2 show the values of $t_{s}$ depending on the target level $x_{T}$. Figure 3 shows the $x_{d}^{*+}$-run connected with the $x_{d}^{*-}$-run at time $t_{s}$. Representative runs of the $x_{t}^{*}$-process determined by Eqs. (19) and (20) emanating from $x_{0}=1$ and $x_{0}=0.5$ to the target states $x_{T}=0.5$ and $x_{T}=1$ are shown in Figs. 4 and 5 , respectively. Table 2 gives numerical versions of the time evolution of $x_{t}^{*}, \widetilde{x}_{s k}, p_{s k}$ corresponding to the experiments shown in Fig. 4.

\section{CONCLUSION}

A method for finding the finite horizon suboptimal control strategy under the LQG performance criterion has been presented for a class of nonlinear stochastic systems. It is well-known that two solutions are found to the optimal control problem within the framework of dynamic programming approach; one gives the stable optimal state trajectory and the other the unstable one which is turned down the advent.

In this paper, reviving the unstable state trajectory so as to hit the target state and connecting this with the stable optimal state trajectory emanating from the initial state at time $t_{s}$, the suboptimal state trajectory is completed for the control interval $[0, T]$. An inescapable fact is that a discontinuity at time $t_{s}$ occurs on the suboptimal stochastic state trajectories. This fact and a modification of the definition of admissible control will be described in the subsequent paper, including extended versions to the $n$-dimensional stochastic nonlinear system.

\section{REFERENCES}

[1] D. A. Carlson, A. B. Haurie and A. Leizarowitz: Infinite Horizon Optimal Control Deterministic and Stochastic System, Springer, N. Y. 1991.

[2] Y. Sunahara, H. Ohtagaki, T. Tsutsumi: On the control performance and optimal stationary 
states of nonlinear systems, SICE'93, Kanazawa, $369 / 370$.

\section{Appendix-A Derivation of Eq. (6).}

Consider the averaged process, $E_{x_{0}}\left\{x_{t}\right\}=\tilde{x}_{t}$, determined by

$$
\begin{aligned}
& \frac{d \tilde{x}_{t}}{d t}=f\left(\tilde{x}_{t}\right)+b \tilde{u}_{t}, \quad \tilde{x}(0)=\tilde{x}_{0} \\
& \text { for } \quad 0 \leq t<\infty,
\end{aligned}
$$

where $\tilde{u}_{t}=E_{x_{0}}\left\{u_{t}\right\}$ and an approximation $\tilde{f}\left(x_{t}\right)=$ $f\left(\tilde{x}_{t}\right)$ has been made. The performance criterion is

$$
\min _{\tilde{u}_{t} \in \mathcal{U}^{0}}\left[\tilde{J}_{T}\left(\tilde{u}_{t}\right)=\int_{0}^{\infty}\left\{L_{s}\left(\tilde{x}_{t}\right)+n \tilde{u}_{t}^{2}\right\} d t\right],
$$

where

$$
L_{s}\left(\tilde{x}_{t}\right)=m \tilde{x}_{t}^{2}+\frac{1}{2} \sigma_{0} \tilde{x}_{t}^{2} V_{\tilde{x}_{t} \tilde{x}_{t}} .
$$

The Hamilton-like function associated with (A.1) and its stationary version are

$$
\begin{aligned}
\tilde{K}\left(\tilde{x}_{t}, \tilde{u}_{t}, \tilde{V}_{\tilde{x}_{t}}\right)=m \tilde{x}_{t}^{2}+n \tilde{u}_{t}^{2} \\
+\left\{f\left(\tilde{x}_{t}\right)+b \tilde{u}_{t}\right\} V_{\tilde{x}_{t}}+\frac{1}{2} \sigma_{0}^{2} \tilde{x}_{t}^{2} V_{\tilde{x}_{t} \tilde{x}_{t}}
\end{aligned}
$$

and

$$
\tilde{K}_{s t}\left(\tilde{x}_{s t}, \tilde{u}_{s t}\right)=m \tilde{x}_{s t}^{2}+n \tilde{u}_{s t}^{2}+\frac{1}{2} \sigma_{0}^{2} \tilde{x}_{s t}^{2} V_{\tilde{x}_{s t} \tilde{x}_{s t}},
$$

where as far as the control interval of concern is for $t \in[0, \infty), \lim _{t \rightarrow \infty} V_{\tilde{x}_{t} \tilde{x}_{t}}=V_{\tilde{x}_{s t} \tilde{x}_{s t}}$ is allowed to introduce. Differentiating the RHS of (A.5) with respect to $\tilde{u}_{s t}$ and setting the result as zero obtains

$$
\frac{n}{b^{2}} f(\bar{x}) \frac{\partial f(\bar{x})}{\partial \bar{x}}+\frac{1}{2} \sigma_{0}^{2} \bar{x} V_{\bar{x} \cdot \vec{x}}+m \vec{x}=0,
$$

where through $\tilde{x}_{s t}^{*}$ satisfying the condition, $d \tilde{K}_{s t} / d \tilde{u}_{s t}$ $=0$, we define

$$
\lim _{t \rightarrow \infty} \tilde{x}^{*}(t)=\tilde{x}_{s t}^{*}=\tilde{x} .
$$

In the LQG performance criterion, it follows that [2]

$$
V_{\vec{x} x}=2 p_{s} .
$$

Substitution of (A.8) into (A.6) brings (6)

Appendix-B Derivation of Eq. (7).

The condition $d K / d u_{t}=0$ in Eq. (5) is

$$
u_{t}^{*}=-\frac{b}{2 n} V_{x_{i}^{*}},
$$

where we write $u_{t}^{*}, x_{t}^{*}$ satisfying the condition for $u_{t}, x_{t}$, respectively. Then,

$$
\begin{aligned}
K^{*}\left(x_{t}^{*}, V_{x_{i}^{*}}, V_{x_{i}^{*} x_{i}^{*}}\right)=m x_{t}^{* 2}-\frac{1}{4} \frac{b^{2}}{n} V_{x_{i}^{*}}^{2} \\
+f\left(x_{t}^{*}\right) V_{x_{i}^{*}}+\frac{1}{2} \sigma_{0}^{2} x_{t}^{* 2} V_{x_{i}^{*} x_{i}^{*}}
\end{aligned}
$$

Applying (B.1) to Eq. (1) obtains,

$$
V_{x_{i}^{*}}=\frac{2 n}{b^{2} d t}\left[f\left(x_{t}^{*}\right) d t+\sigma_{0} x_{t}^{*} d w_{t}-d x_{t}^{*}\right] .
$$

On the other hand, noting that $f\left(\tilde{x}_{s t}\right)+b \tilde{u}_{s t}^{*}=0$,

$$
\begin{aligned}
& \tilde{K}_{s t}^{*}\left(\tilde{x}_{s t}^{*}, \tilde{u}_{s t}^{*}\right) \\
& \quad=m \tilde{x}_{s t}^{* 2}+\frac{1}{2} \sigma_{0}^{2} \tilde{x}_{s t}^{* 2} V_{\tilde{x}_{s t}^{*}}+\frac{n}{b^{2}} f^{2}\left(\tilde{x}_{s t}^{*}\right) .
\end{aligned}
$$

Based on the assumption that

$$
K^{*}\left(x_{t}^{*}, V_{x_{i}^{*}}, V_{x_{i}^{*} x_{i}^{*}}\right)=\tilde{K}_{s t}\left(\tilde{x}_{s t}^{*}, \tilde{u}_{s t}^{*}\right),
$$

from (B.2), (B.3), (B.4), (A.7) and (A.8), after somewhat tedious calculations, Eq. (7) can be derived.

Table 1: The switching time $t_{s}$ vs. $\bar{x}_{d}^{*}(T) \quad\left(x_{0}=\right.$ 1, $T=12$ )

\begin{tabular}{|c|c|c|}
\hline$x_{T}$ & $t_{s}$ & $\bar{x}_{d}^{*}(T)$ \\
\hline 1.00 & 10.810 & 0.9989 \\
\hline 0.75 & 10.953 & 0.7495 \\
\hline 0.50 & 11.093 & 0.4998 \\
\hline 0.25 & 11.308 & 0.2492 \\
\hline
\end{tabular}


Table 2: The numerical versions of time evolution of suboptimal state, linearized steady state and Riccati solution

\begin{tabular}{|c|c|c|c|}
\hline$t$ & $\bar{x}_{s k}$ & $x_{t}^{*}$ & $p_{s k}$ \\
\hline 0 & & 1.0 & \\
\hline 1.0 & $-2.167 \times 10^{-4}$ & $3.740 \times 10^{-2}$ & $-1.607 \times 10^{-1}$ \\
\hline 2.0 & $-2.771 \times 10^{-6}$ & $1.534 \times 10^{-2}$ & $-1.600 \times 10^{-1}$ \\
\hline 3.0 & $-1.773 \times 10^{-6}$ & $1.178 \times 10^{-2}$ & $-1.600 \times 10^{-1}$ \\
\hline 4.0 & $-1.737 \times 10^{-6}$ & $1.795 \times 10^{-2}$ & $-1.600 \times 10^{-1}$ \\
\hline 5.0 & $-1.736 \times 10^{-6}$ & $1.354 \times 10^{-2}$ & $-1.600 \times 10^{-1}$ \\
\hline 6.0 & $-1.736 \times 10^{-6}$ & $1.530 \times 10^{-2}$ & $-1.600 \times 10^{-1}$ \\
\hline 7.0 & $-1.736 \times 10^{-6}$ & $1.439 \times 10^{-2}$ & $-1.600 \times 10^{-1}$ \\
\hline 8.0 & $-1.736 \times 10^{-6}$ & $1.861 \times 10^{-2}$ & $-1.600 \times 10^{-1}$ \\
\hline 9.0 & $-1.736 \times 10^{-6}$ & $1.367 \times 10^{-2}$ & $-1.600 \times 10^{-1}$ \\
\hline 10.0 & $-1.736 \times 10^{-6}$ & $1.651 \times 10^{-2}$ & $-1.600 \times 10^{-1}$ \\
\hline 11.0 & $-1.736 \times 10^{-6}$ & $1.448 \times 10^{-2}$ & $-1.600 \times 10^{-1}$ \\
\hline 11.092 & $-1.736 \times 10^{-6}$ & $1.307 \times 10^{-2}$ & $-1.600 \times 10^{-1}$ \\
\hline 11.093 & $-1.641 \times 10^{-6}$ & $1.308 \times 10^{-2}$ & 6.249 \\
\hline 12.0 & $-8.650 \times 10^{-2}$ & $4.749 \times 10^{-1}$ & 4.799 \\
\hline
\end{tabular}

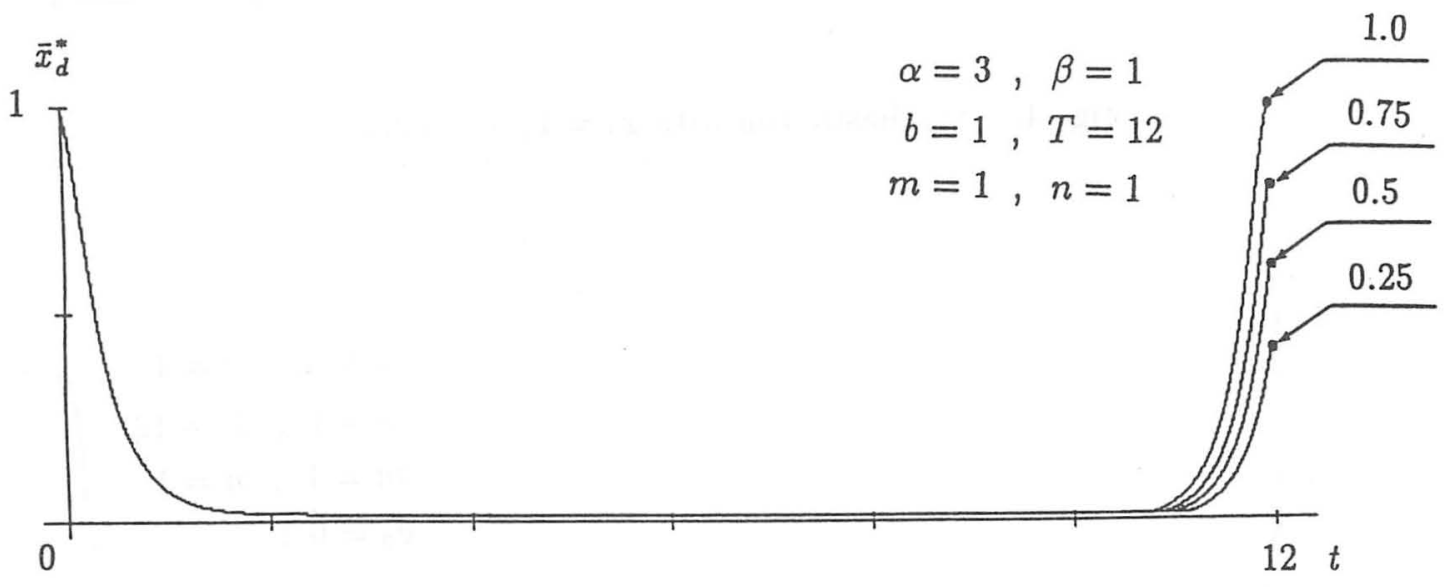

Fig. 2: Deterministic runs hitting various target $x_{T}$ 


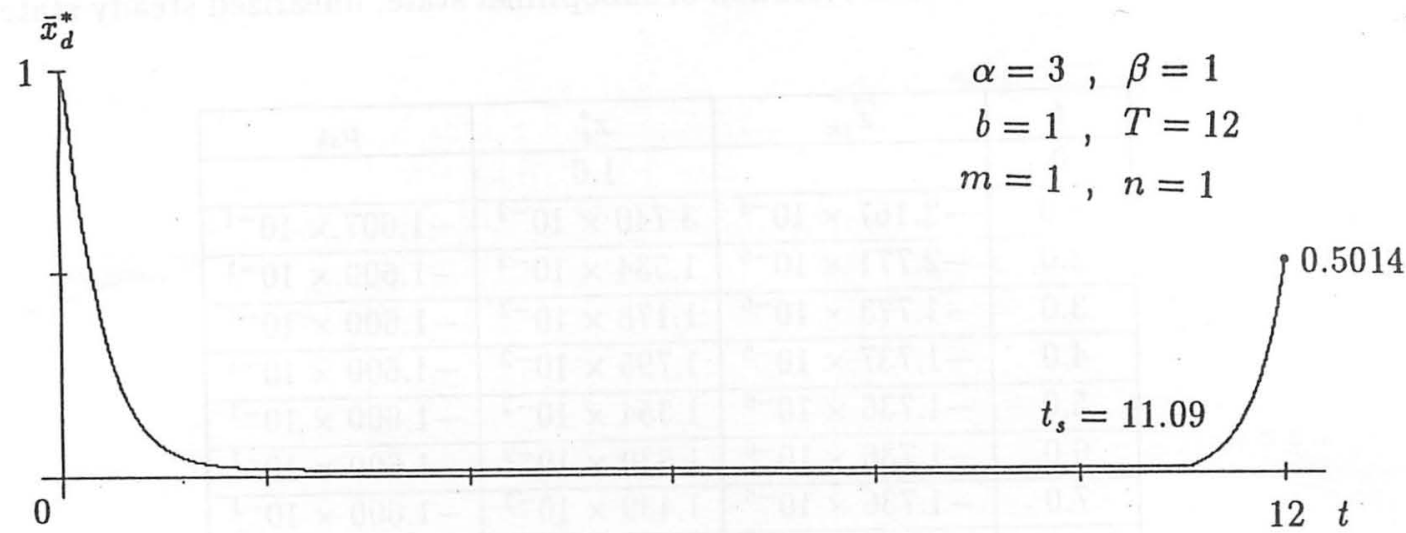

Fig. 3: Deterministic run with $x_{0}=1, x_{T}=0.5$

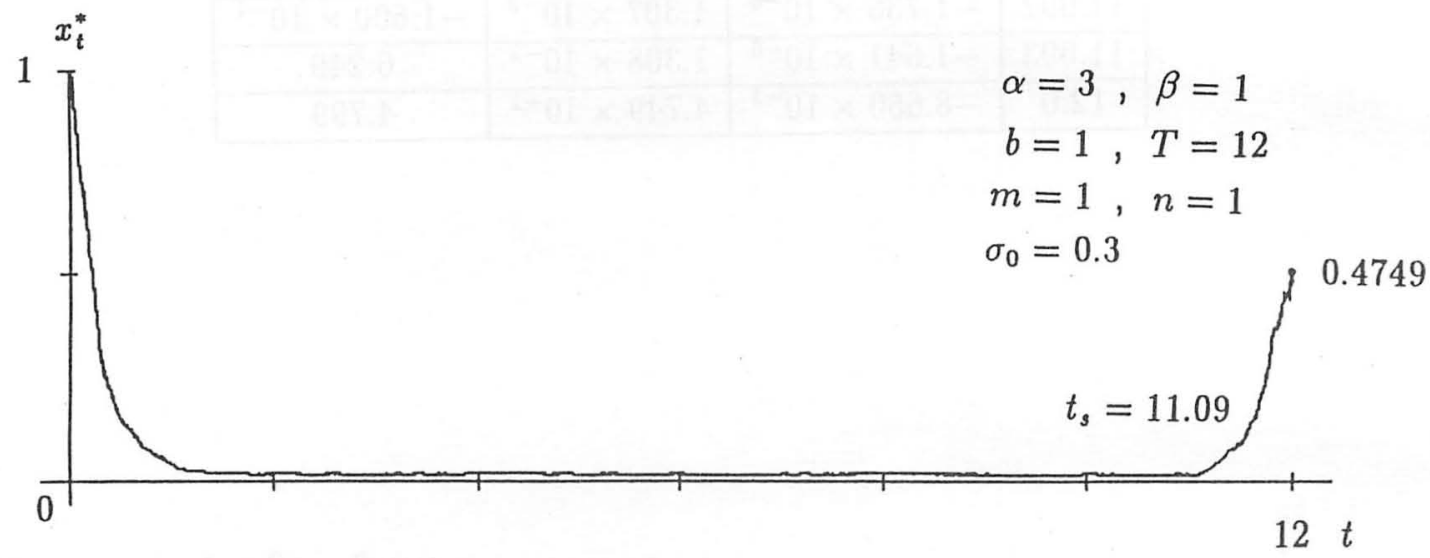

Fig. 4: Stochastic run with $x_{0}=1, x_{T}=0.5$

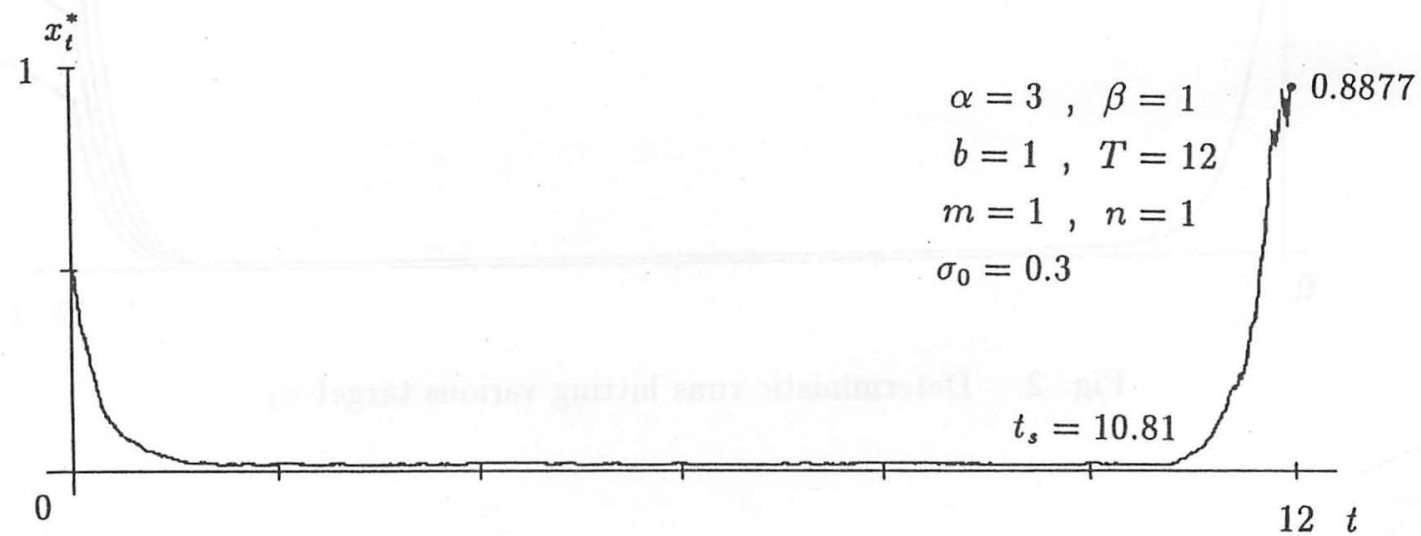

Fig. 5: Stochastic run with $x_{0}=0.5, x_{T}=1$ 